\title{
Traspatios y percepción del programa PESA
}

\author{
Backyards and perception of the PESA program
}

\author{
Erika Román-Montes de Oca ${ }^{*}$, Francisco García-Matías ${ }^{1}$, Manuel de Jesús Sainz-Aispuroํ, \\ Alina Juantorena-Ugás ${ }^{1}$
}

\section{RESUMEN}

Durante años, los traspatios han formado parte de la seguridad alimentaria y son pieza fundamental en las estrategias de vida de las familias rurales. En este contexto, las instituciones han creado modelos basados en los traspatios como una alternativa para promover la seguridad alimentaria; muestra de ello es la implementación del programa Proyecto Estratégico de Seguridad Alimentaria (PESA) en las comunidades marginadas. El objetivo de este trabajo fue describir de qué modo las beneficiarias perciben la contribución del programa PESA en los aspectos económicos y sociales de la unidad familiar. La metodología fue cualitativa a través del método fenomenológico. De un total de 81 mujeres, se encuestó a 40 mujeres y se entrevistó a ocho; la técnica de muestreo utilizada fue la bola de nieve. El resultado fue que $100 \%$ de las encuestadas comentó que el programa mejoró sus ingresos; asimismo, todas ellas dijeron que este apoyo les permitió optimizar el manejo de las aves, beneficiarse en la alimentación y generar una mayor estima.

PALABRAS CLAVE

PESA, mujeres, unidad familiar, estrategia de vida

\section{ABSTRACT}

Backyards have been part of food security for many years. They are a fundamental piece in life strategies for rural family unity. Institutions have considered this model as an alternative to food security. The implementation of the Proyecto Estratégico de Seguridad Alimentaria (PESA) project in marginalized communities is an example. The objective of this work was to determinate how PESA beneficiaries perceives its contribution to economic and social aspects in the family unity. A qualitative method was used, applying a phenomenological techniche; two main poll samples were required. The first one was applied to 40 women, and the second one to 8 of 81 cases. In both cases we used the snowball procedure. $100 \%$ of surveyed subjects declared that PESA project increases their incomes. They also said the program had benefits increasing their birds management knowledge, improving food diets, and consolidate their self-esteem.

KEYWORDS

PESA, women, family unity, life strategies

\footnotetext{
${ }^{1}$ Facultad de Ciencias Agropecuarias, Universidad Autónoma del Estado de Morelos. Cuernavaca, México.

* Autor para correspondencia. Av. Universidad 1001, col. Chamilpa. 62209 Cuernavaca, Morelos, México. Correo electrónico: erika.romanm@ uaem.edu.mx
} 


\section{INTRODUCCIÓN}

A lo largo de los años, instituciones nacionales e internacionales se han preocupado por la reducción del hambre y se han programado metas para erradicarla. Sin embargo, los programas implementados no han beneficiado ni disminuido la pobreza ni el hambre de las poblaciones, debido a que han desempeñado un papel para la asistencia social y no para reactivar las economías locales (FAO et al., 2015; Lobo, 2014).

En la década de los setenta se dio el auge de la producción agropecuaria: se apostó a la productividad extensiva con monocultivos, surgieron centros de investigación para implementar técnicas y tecnologías modernas y se benefició a los productores, quienes aceptaron el cambio en los procesos productivos; todo esto con la intención de incrementar la producción nacional y fomentar que los campesinos se convirtieran en empresarios. Sin embargo, como ya muchos autores lo han planteado (Bonfil, 1991; Chiriboga, 1997; Román, 2016), estos procedimientos no tomaron en cuenta que la gente de las comunidades rurales tiene su propia forma de producir -la cual suele variar de una comunidad a otra-, que cada lugar tiene su particular historia, identidad y costumbres pero, sobre todo, que su mentalidad es diferente a la de los empresarios capitalistas. La gente del campo, los campesinos, siembran para comer, para vivir, para convivir y para recordar, no sólo para obtener ganancia económica.

Por lo anterior, aunque se hayan realizado esfuerzos por implementar técnicas y tecnologías "modernas", muchos campesinos continúan sembrando de forma tradicional, porque este método es amigable con el medio ambiente, las tradiciones, la identidad y, sobre todo, la seguridad alimentaria. Éste es un derecho que por ley les corresponde, según el artículo $4^{\circ}$ de la Constitución Política de los Estados Unidos Mexicanos, el cual dice que "Toda persona tiene derecho a la alimentación nutritiva, suficiente y de calidad. El Estado lo garantizará" (Carbonell, 2015). La seguridad alimentaria existe cuando "todas las personas tienen en todo momento acceso físico y económico a suficientes alimentos inocuos y nutritivos para satisfacer sus necesidades alimenticias y sus preferencias en cuanto a los alimentos a fin de llevar una vida activa y sana" (FAO, 1996). Además, el acceso a la seguridad alimentaria implica cumplir con sus cuatro dimensiones: la disponibilidad física de los alimentos, el acceso económico y físico a los alimentos, la utilización de los alimentos y la estabilidad (FAO, 2011; IICA, 2012).
La Organización de las Naciones Unidas para la Agricultura y la Alimentación (FAO), junto con los gobiernos de diferentes países latinoamericanos, buscó alternativas para mitigar el hambre en el mundo. Una de ellas fue el Proyecto Estratégico de Seguridad Alimentaria (PESA), que se lleva a cabo en México en coordinación con la Secretaría de Agricultura, Ganadería, Desarrollo Rural, Pesca y Alimentación (SAGARPA). Se trata de una estrategia que, desde 2002, ha aportado esfuerzos para transformar las condiciones de vida en comunidades rurales de marginación alta y muy alta en el país. El objetivo de este proyecto es "contribuir al desarrollo de capacidades de las personas y familias que se encuentran en comunidades de alta marginación, para incrementar la producción agropecuaria, innovar los sistemas de producción, desarrollar los mercados locales, promover el uso de alimentos y la generación de empleos para lograr su seguridad alimentaria y el incremento en el ingreso" (PESA-Sagarpa, 2017).

En el estado de Morelos, el proyecto PESA se implementó a partir de 2008 y, desde entonces, ha buscado asegurar la alimentación de las unidades de producción familiar que participan en el programa, promoviendo elevar la motivación de las familias y las comunidades rurales a través de la producción de alimentos y desarrollo de capacidades para mejorar la disponibilidad y el acceso a éstos.

Uno de los principales apoyos que otorga este programa es para la producción de huevo de gallina. Debemos recordar que esta ave se encuentra en casi todos los traspatios de México; es la más común porque de ella se consumen tanto el huevo como la carne, como lo mencionan diferentes estudios relacionados con los traspatios (Cruz et al., 2016; González et al., 2014; Verduzco et al., 2016). Esta producción no requiere importantes manejos, pero sí otorga grandes beneficios; por ello, las familias de las comunidades rurales continúan ejerciéndola.

Se han realizado diversos estudios relacionados con la contribución del programa PESA en diferentes estados del país; sin embargo, no se ha investigado específicamente sobre la percepción que tienen los beneficiarios respecto a esta contribución. Los trabajos que se indagaron tienen como objetivo principal la producción y los beneficios de la seguridad alimentaria de las unidades domésticas beneficiadas.

Gimate (2016) realizó un análisis basado en la evidencia de seguridad alimentaria de diferentes comunidades del estado de Oaxaca y encontró que los beneficiarios percibían que el programa PESA influía en la seguridad alimentaria de las unidades 
domésticas debido a que tienen mejor disponibilidad, acceso y uso de los alimentos. También encontró que $90 \%$ de los beneficiarios adoptó algunos proyectos porque había obtenido mejoras en la cría de aves y producción de huevo.

Sin embargo, otro estudio realizado en Oaxaca por Verduzco et al. (2016) menciona que los proyectos avícolas promovidos por el PESA tienen sistemas semi-intensivos de doble propósito, con parvadas de 12 aves en edad reproductiva en promedio y con parámetros productivos y reproductivos pobres, por lo que contribuyen muy poco a la seguridad alimentaria o no representan una fuente competitiva de ingresos para los beneficiarios de dicho programa.

Por otra parte, Martínez et al. (2013) investigaron que, de los proyectos de caprinos apoyados con el programa PESA, $37.3 \%$ se encuentra decreciendo o cerrado, y que $62.7 \%$ se mantiene o crece. El nivel de producción y crecimiento de los rebaños es marginal; por lo tanto, hasta el momento no han sido una opción de ingreso, alimentación o capitalización. Actualmente, la operación de estos proyectos no ofrece atractivos económicos y acentúa la sobrecarga de los agostaderos.

En lo que respecta a la avicultura de traspatio, la estrategia que busca el programa PESA es la contribución a la autosuficiencia de los beneficiarios $\mathrm{y}$, por consiguiente, de las comunidades. Cruz et al. (2016), en su estudio, encontraron que los resultados sugieren que los estatus "deseables" están asociados a unidades avícolas de producción familiar (UAPF) que destinan mayores volúmenes de su producción a la venta. Es decir, que el incentivo de generar ingresos para la familia es fundamental para la permanencia de los proyectos.

La evaluación del programa PESA es variada y depende de diversos factores y perspectivas de cada comunidad. Ha permanecido durante años y trata de otorgar las herramientas necesarias para proporcionar seguridad alimentaria a las unidades domésticas de las comunidades marginadas. En Morelos, este aspecto no se ha estudiado a profundidad.

En este contexto, el objetivo de este estudio fue determinar cómo perciben las beneficiarias la contribución del programa PESA de dos comunidades rurales de Morelos, México, en los aspectoseconómicos y sociales de la unidad familiar. La hipótesis es que el apoyo otorgado a través del programa beneficia a las familias en la producción de los traspatios, ya que genera alimento para autoconsumo, así como un ingreso extra para cubrir las necesidades básicas que éstas no producen. Además, beneficia a las mujeres porque les genera mayor confianza en sí mismas.

\section{Materiales y MÉtodos}

El trabajo de investigación se realizó en las comunidades de Huitchila y Zacapalco, pertenecientes al municipio de Tepalcingo, Morelos, México. El municipio se localiza entre los paralelos $18^{\circ} 35^{\prime}$ de latitud norte y $98^{\circ} 50^{\prime}$ de longitud oeste del meridiano de Greenwich, a una altura de 1,160 msnm. Los municipios vecinos son: al norte, Ayala y Jonacatepec; al sur, Tlaquiltenango y el estado de Puebla; al este, Axochiapan y Jonacatepec y, al oeste, Ayala y Tlaquiltenango. Cuenta con una superficie de $367.67 \mathrm{~km}^{2}$, cifra que representa $7.05 \%$ del total del estado. Su población es de 12,053 habitantes: 5,796 hombres y 6,257 mujeres (INEGI, 2010). La superficie sembrada es de 7,346 ha y la cosecha es de 7,327 ton. En 2015, la producción fue de 544 ton de bovinos, 941 ton de porcinos, 55 ton de ovinos, 53 ton de caprinos, 3,769 ton de aves y 17 ton de huevo para plato (SIAP, 2015).

La comunidad de Huitchila cuenta con 958 hombres y 977 mujeres. El grado promedio de escolaridad es de 6.67 años: 6.37 para las mujeres y 6.96 para los hombres. La población económicamente activa es de 730: la masculina es de 539 y la femenina, de 191. El índice de fecundidad es de 3.03 hijos por mujer. El total de viviendas es de 494, de las cuales 395 tienen jefatura masculina y 99, femenina (INEGI, 2010).

La localidad de Zacapalco tiene 1,714 habitantes, de los cuales 813 son hombres y 901 son mujeres. El grado promedio de escolaridad es de 6.43 años: 6.86 para las mujeres y 5.97 para los hombres. La población económicamente activa es de 603: 443 la masculina y 160 la femenina. El índice de fecundidad es de 2.97 hijos por mujer. El total de viviendas es de 435 , de las cuales 298 tienen jefatura masculina y 137, femenina (INEGI, 2010).

El trabajo de investigación se llevó a cabo a través del enfoque cualitativo que, según Hernández et al. (2006), se define como un conjunto de prácticas interpretativas que hacen al mundo visible; lo transforman y convierten en una serie de representaciones en forma de observaciones, anotaciones, grabaciones y documentos. Este enfoque es asimismo naturalista (porque estudia a los objetos y seres vivos en sus contextos o ambientes naturales) e interpretativo (pues intenta encontrar sentido a los fenómenos en términos de los significados que las personas les otorguen).

El método que se utilizó fue el fenomenológico, que es el estudio de la experiencia vital, del mundo de la vida, de la cotidianidad. Lo cotidiano, en sentido fenomenológico, es la experiencia no conceptualizada o categorizada. Asimismo, este método 
ha hecho diversas aportaciones a la investigación cualitativa: la primacía que otorga la fenomenología a la experiencia subjetiva inmediata como base para el conocimiento; el estudio de los fenómenos desde la perspectiva de los sujetos, teniendo en cuenta su marco referencial, y su interés por conocer cómo las personas experimentan e interpretan el mundo social que construyen en interacción. Por lo tanto, el fenomenólogo intenta ver las cosas desde el punto de vista de otras personas, describiendo, comprendiendo e interpretando (Rodríguez et al., 1996).

La muestra se obtuvo con la técnica de "bola de nieve", que consiste en solicitar a los informantes que recomienden a posibles participantes. Esta técnica fue seleccionada porque, gracias a la presentación que hace el sujeto ya incluido en el proyecto, resulta más fácil establecer una relación de confianza con los nuevos participantes. También permite acceder a personas difíciles de identificar; además de que el investigador tiene menos problemas para especificar las características que desea de los nuevos participantes. Este proceso de selección se apoya en las redes sociales naturales (Monje, 2011).

Para esta investigación, se identificó a las mujeres que fueron apoyadas con proyectos para módulos de gallinas del programa PESA en 2012, 2013 y 2014, cuyas aportaciones fueron agregadas a la muestra. Se les preguntó si conocían a otras personas que pudieran proporcionar datos $\mathrm{y}$, una vez que facilitaban el nombre de una tercera, se procedía a encuestarla o entrevistarla, y así sucesivamente hasta que se obtuvo una muestra representativa de $52 \%$ en Zacapalco y $48 \%$ en Huitchila.

De un total de 81 beneficiarias (42 en Zacapalco y 39 en Huitchila), se aplicaron 40 encuestas: 20 en Zacapalco y 20 en Huitchila. El cuestionario consiste en un formato que se llena de forma escrita. Esta técnica disminuye los sesgos ocasionados por la presencia del entrevistador; además, facilita el análisis y reduce los costos de aplicación (Monje, 2011). Asimismo, se realizaron ocho entrevistas semiestructuradas a las beneficiarias del programa de las dos comunidades. Este tipo de entrevistas siguen la propuesta de Álvarez (2003) y se enfocan en entender la perspectiva del entrevistado así como en recolectar sus impresiones y experiencias en relación con el tema que se estudia (Landini, 2012). Se utilizaron cuatro rubros y 33 reactivos (anexo 1), que se clasificaron de la siguiente manera:

Rubro 1. Características del entrevistado: 5 reactivos. Rubro 2. Socioeconómico: 7 reactivos.

Rubro 3. Traspatio: 4 reactivos.

Rubro 4. Apoyo PESA: 19 reactivos.
El trabajo de campo se hizo en el periodo comprendido entre agosto y noviembre de 2016. También se realizaron recorridos de campo en las comunidades, así como observación de participantes e informantes clave. La información se sistematizó y analizó mediante una base de datos con el programa Excel@, a partir del cual se obtuvieron datos descriptivos que permitieron visualizar el objetivo y la hipótesis del trabajo. Se hizo una revisión bibliográfica y se consultaron bases de datos en medios electrónicos, con la finalidad de conocer la evaluación del programa PESA en diferentes estados del país, en relación con la percepción de los beneficiarios en los aspectos de seguridad alimentaria, económicos y sociales, así como de conocer el número de beneficiarios de proyectos de producción de huevo de traspatio o producción de aves del programa PESA en las comunidades de estudio.

\section{RESULTADOS Y DISCUSIÓN}

El tema de la seguridad alimentaria ha sido muy importante en el discurso oficial durante varias décadas. Sin embargo, no fue sino hasta 1996 cuando las instituciones internacionales y nacionales de los diferentes países empiezan a preocuparse más, lo que las motivó a generar una serie de estrategias para que hubiera acceso a los alimentos, principalmente a través de la reactivación de programas que beneficien la producción familiar a pequeña escala (FAO et al., 2015; González, 2007; IICA, 2012; Oseguera y Esparza, 2009). En muchos países, la producción de alimentos se ha ido incrementando con los años al igual que la desnutrición de los habitantes. Esta paradoja se debe a que uno de los principales problemas que enfrentan las familias es el acceso a los alimentos nutritivos.

Dado que las empresas internacionales tienen el dominio del mercado y que en muchas ocasiones lo que buscan es el beneficio económico a costa de lo que sea -aun en perjuicio de miles y millones de habitantes-, es ineludible que los países importadores de alimentos, los que dependen de otras naciones para tener seguridad alimentaria, apuesten cada día más a la producción interna de alimentos.

Un ejemplo de esta problemática se documentó en los estudios realizados por Houtart (2010) sobre los agrocarburantes: la expectativa de los intereses económicos y políticos de estos productos no parece ser una solución a la crisis energética ni a la climática; más bien se deja ver como una amenaza al medio ambiente, en particular de los países del Sur de América; éste es un peligroso factor del agravamiento de la crisis alimentaria, pero principalmente, una salida muy 


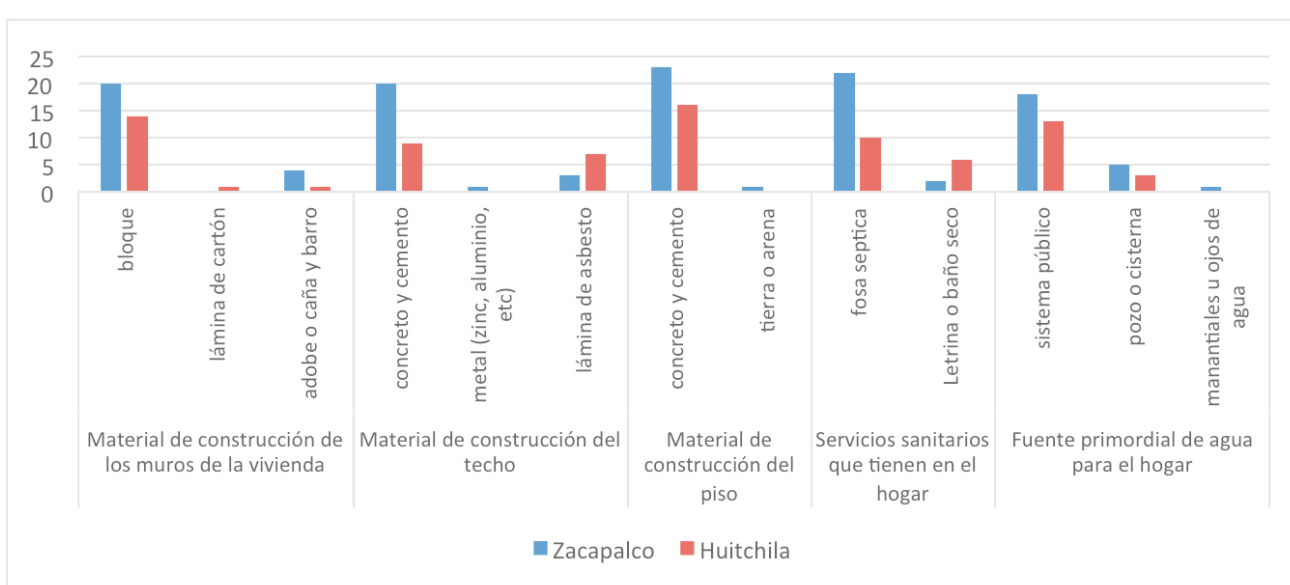

Figura 1. Servicios y materiales de construcción de las viviendas de las encuestadas.

rentable para la crisis del capital y con unos colosales costos sociales y políticos concretamente en los países subdesarrollados. Obidzinski et al. (2012) evaluaron los impactos ambientales y socioeconómicos de las plantaciones de palma asociadas con la producción de bioenergía en Indonesia y encontraron que las tierras degradadas son causa de la subvaloración de los bosques y del hecho de haberlas considerado un activo económico. Ordoñez (2010), en un estudio sobre la agroecología y la soberanía alimentaria como alternativas al sistema agroalimentario capitalista, comenta que el problema del hambre se ha identificado no como un problema de producción de alimentos, sino como un problema político y económico, en el cual el capitalismo se orienta cada vez más a la generación de riqueza por encima de imperativos sociales y ambientales. Riat y Pochettino (2014) reflejan un análisis comparativo entre dos modelos de producción: uno netamente extractivo y otro de uso sustentable de los bienes comunes. Rubio (2013) hace referencia a los beneficios obtenidos por las empresas transnacionales y las instituciones gubernamentales, sobre las crisis económicas en México.

Sin embargo, a pesar de todos los cambios de vida y arbitrariedades de estos organismos, a través de las décadas, las personas de las comunidades rurales se van adaptando y buscan diferentes actividades para tener seguridad alimentaria. Un actor importante en este tema es, precisamente, la mujer. Como lo mencionan Espinosa y Diez-Urdanivia (2006); Guzmán (2005); Román y Guzmán (2013) es una administradora nata, es la responsable vital del bienestar de la familia, es quien se hace cargo del huerto familiar, elabora la comida, comercializa localmente, entre muchas otras actividades. Por ello, en este trabajo se habla de la opinión de la mujer sobre los apoyos para módulos de gallinas del programa PESA, ya que éste la considera un actor principal en la seguridad alimentaria de la unidad doméstica: la mujer tiene un papel fundamental en la alimentación, se preocupa más que el varón respecto a tener alimentos en la mesa, es quien aporta en mayores proporciones recursos para la alimentación y, en la mayoría de las ocasiones, es quien se encarga del traspatio.

\section{Mujeres y el traspatio}

La mayoría de las personas encuestadas vive en la localidad desde hace más de 21 años (98\%); 41\% oscila entre 41 y 60 años de edad, $24 \%$ tiene menos de 40 años y 17\%, más de 60; lo anterior muestra la permanencia de las familias en las comunidades. Asimismo, 93\% cuenta con casa propia, mientras que el resto habita en una vivienda rentada o prestada. El hecho de que la mayor parte posea una casa, les permite construir y plantar con mayor facilidad lo requerido en el traspatio. De acuerdo con los datos socioeconómicos que proporcionaron, se pudo observar que la generalidad de las encuestadas de la comunidad de Huitchila cuenta con menos servicios y que el material de construcción de las casas donde habitan es de menor calidad (figura 1).

En el trabajo de observación y las entrevistas realizadas, se apreció que Huitchila tiene una actividad agropecuaria más desarrollada en comparación con Zacapalco. Las unidades domésticas reciben varios apoyos por parte de diferentes instituciones gubernamentales encaminados hacia el desarrollo humano, cuyo principal requisito es que los beneficiarios sean familias de escasos recursos; por lo tanto, deben mantenerse en condiciones físicas visuales de pobreza para poder tener acceso a los apoyos, aunque, por 
otro lado, prefieren invertir el recurso excedente en las actividades relacionadas con la producción agropecuaria. En Zacapalco se presentan las mismas características; no obstante, de acuerdo con las familias entrevistadas, en esta comunidad es mayor el número de migrantes a Estados Unidos, quienes envían recursos para la producción agropecuaria y para mejorar la infraestructura de las viviendas. Los servicios de luz y recolección de basura son proporcionados por el ayuntamiento y en todos los hogares mencionaron contar con ellos.

En cuanto a la escolaridad, 52\% estudió hasta la secundaria, 34\% cursó la primaria, 9\% mencionó no tener estudios y solamente $5 \%$ dijo haber estudiado el bachillerato (anexo 2). Por lo tanto, en ambas comunidades la mayor parte de las beneficiarias saben leer y escribir; ésta es una ventaja que tienen en comparación con algunos habitantes de comunidades marginadas, quienes no tuvieron oportunidad de asistir a la escuela y cuya vida es aun más difícil; por ejemplo: Ahuaxtla de Axochiapan, Hueyapan de Tetela del Volcán y Xoxocotla de Puente Ixtla (INEGI, 2010). Tener limitada la capacidad de leer y escribir significa pertenecer a un grupo social de alto riesgo, en términos de pobreza y marginación (Suárez, 2001).

$\mathrm{Al}$ saber leer y escribir tienen mayores oportunidades de crecer, participar en diferentes actividades y ser menos dependientes; comolo refieren los estudios realizados por Adebayo y Adeola (2005), Emaikwu et al. (2011) y Salah et al. (2017), quienes señalan que el nivel educativo de los productores ha tenido una relación positiva y significativa con la producción. Asimismo, en la investigación de Román y Guzmán (2013), realizada en la comunidad de Amatlán de Quetzalcóatl, Tepoztlán, Morelos, manifiestan que la educación para los habitantes de la comunidad es un elemento considerado fundamental en la preparación y la posibilidad de una mejor vida. Actualmente, las madres están motivando a sus hijas a que estudien niveles educativos superiores a la primaria. Las mujeres de mayor y mediana edad, entre 40 y 60 años, son las que han tenido menos oportunidades escolarizadas y han experimentado más las carencias del medio rural.

El promedio de integrantes por familia es de cuatro y el ingreso de la unidad familiar por mes oscila aproximadamente en $\$ 3,500$ (187 dólares americanos). Las encuestadas mencionaron que todas son amas de casa; solamente una dijo que es trabajadora y otra, comerciante; sin embargo, señalan que en el hogar realizan muchas actividades que son propias del cuidado de la unidad familiar, como aseo de la casa, cuidado de los hijos, elaboración de alimentos, cuidado del traspatio, apoyo en actividades de la parcela, venta de algunos productos que se obtienen del traspatio o parcela, actividades en la escuela y comunidad, principalmente.

Los traspatios son una unidad productiva que aporta una gran diversidad de alimentos, por lo que es importante hacer énfasis en la producción de especies vegetales y animales a través de las necesidades familiares y del mercado, con el propósito de favorecer la reducción de la pobreza, inseguridad alimentaria y contribuir a la salud de los grupos domésticos (Bonilla et al., 2013; González et al., 2014; Salah et al., 2017).

Asimismo, la producción de vegetales, la colecta de frutas y la cría de animales de traspatio "representa, para las unidades domésticas, caja de ahorro debido a que en situaciones difíciles estos productos adquieren valor de cambio para hacer frente a algunos problemas familiares" (Guarneros et al., 2014; Monroy et al., 2016; Salazar et al., 2015). Otro elemento importante del traspatio es el estético "que representa el cultivo de plantas ornamentales" (López et al., 2013).

Dentro de los hogares de las encuestadas se encuentra el traspatio. En éste tienen diversas plantas, entre las que destacan: albahaca (Ocimum basilicum L.), romero (Rosmarinus officinalis L.), rosas (Rosa sp.), epazote (Dysphania ambrosioides (L.) Mosyakin \& Clemants), pápalo (Porophyllum ruderale (Jacq.) Cass.), cilantro (Coriandrum satioum L.), rábano (Raphanus sativus L.), hierbabuena (Mentha spicata L.), calabaza (Cucurbita pepo L.), chile (Capsicum annuum L.), chayote (Sechium edule (Jacq.) Sw.), bambú (Bambusa spp), caña (Saccharum officinarum L.), árboles de: limones (Citrus limon (L.) Osbeck), guayabo (Psidium guajava L.), guaje (Leucaena leucocephala (Lam.) de Wit), mandarino (Citrus nobilis Lour.), naranjos (Citrus sinensis (L.) Osbeck), maracuyá (Passiflora edulis Sims), nanche (Byrsonima crassifolia (L.) Kunth), aguacate (Persea americana Mill.), guamúchil (Pithecellobium dulce (Roxb.) Benth), mango (Mangifera indica L.), ciruelo (Prunus domestica L.), granado (Punica granatum L.), papayo (Carica papaya L.), guanábano (Annona muricata L.), durazno (Prunus pérsica (L.) Batsch) y lima (Citrus aurantifolia (Christm.) Swingle). Asimismo, tienen animales como: gallinas (Gallus gallus L. subsp. domesticus), borregos (Ovis aries L.), bovinos (Bos Taurus L.), guajolotes (Meleagris gallopavo L.), burros (Equus asinus L.), codornices (Coturnix coturnix (L.)), cerdos (Sus scrofa L. subsp. domestica) y yeguas (Equus caballus L.).

El traspatio permite suministrar alimentos a la unidad familiar y les facilita realizar actividades que 
les proporcionan ingresos: "me ayuda porque me mantiene ocupada y al mismo tiempo cuando necesito dinerito vendo algo y ya con eso puedo comprar otras cosas" (Martina Tepexpa Sánchez, comunicación personal, 2016).

Colín et al. (2012) y López et al. (2013) expresan que los productos obtenidos en el traspatio contribuyen a la alimentación de la familia, al ahorro en la compra de alimentos y a la obtención de ingresos por la venta de algunos productos, así como a la generación de insumos que se utilizan en el mismo traspatio; todo ello se configura en una serie de ahorro.

Este sistema de producción constituye asimismo un lugar de recreación y convivencia: sustenta los lazos familiares y permite realizar diversas actividades durante todo el año, durante el cual se convierte en el sitio preferido de los niños para jugar, escuchar las anécdotas e historias que cuentan las madres o abuelos; aquí las mujeres bordan, cosen, lavan o cocinan; en días de descanso se reúne la familia para comer, platicar o recibir visitas (Guzmán, 2004; Vieyra et al., 2004). Ejemplo de lo anterior es el siguiente testimonio: "cuando llega mi marido y está haciendo mucho calor nos sentamos aquí afuera para refrescarnos y platicar un poco, también mis hijos vienen y se sientan, a veces estamos comiendo fruta de los árboles" (Jovita Riquemel Trejo, comunicación personal, 2016).

Las familias tienen plantas aromáticas $\mathrm{y}$ medicinales que utilizan para elaborar la comida o para prevenir o curar algunas enfermedades; los remedios caseros continúan utilizándose por tradición y para disminuir el costo de las consultas o el tiempo que les lleva conseguir una consulta (González et al., 2014; Guerrero, 2007), como deja ver el comentario de Patricia: "cuando alguien de la familia se siente enfermo, le duele la panza o está agripado, le hago un té de epazote o hierba buena o según lo que le duela es la planta que escojo. Muchas veces ayuda y otras, cuando no, lo tenemos que llevar al centro de salud" (Patricia Sánchez Riquemel, comunicación personal, 2016).

En relación con la participación en el traspatio, se pudo valorar que, en la comunidad de Zacapalco, en $28 \%$ de las unidades familiares, todos los integrantes de la familia participan en el cuidado del traspatio; a su vez, en Huitchila, en $25 \%$ de los casos, la esposa es la única en cuidarlo y mantenerlo. Con menores porcentajes están el jefe de familia, los hijos, la esposa e hijos, adulto mayor, esposa y el jefe de familia (figura 2).

Sin embargo, en la mayoría de los casos, las mujeres participan en el cuidado del traspatio porque

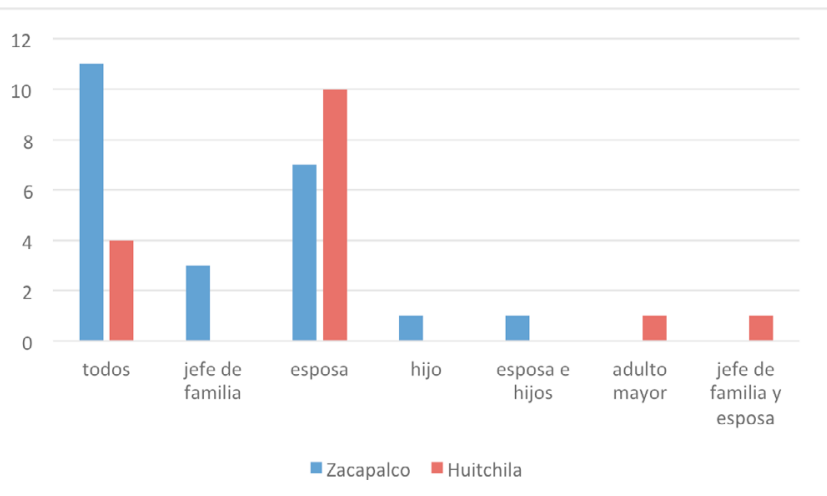

Figura 2. Participación de los integrantes de la unidad familiar en el traspatio.

se considera como parte de las actividades del hogar; son las responsables principales de mantenerlo en condiciones óptimas de producción, se encargan del cuidado de los animales, las plantas, la limpieza y demás actividades requeridas. Esto coincide con los hallazgos de Vieyra et al. (2004), según los cuales generalmente la mujer se encarga de los asuntos económicos de la casa, del abastecimiento de los víveres, de conocer los precios en el mercado de los productos que son ocupados en el traspatio y de la venta de la producción. Además, contribuye al medio ambiente y es la trasmisora a los hijos e hijas de conocimientos relacionados con el cuidado de la naturaleza para la obtención de recursos.

La mujer es la que se ocupa de la alimentación y la salud de la unidad doméstica; por lo tanto, conoce qué es lo que se requiere tener en el traspatio. Lo anterior está de acuerdo con Salah et al. (2017), quienes indican que las mujeres casadas comúnmente están involucradas en la producción de pollos para obtener su propia carne de ave y huevos. Asimismo, Salazar et al. (2015) reportaron la relevancia de la participación de la mujer en el cuidado del traspatio.

Por su parte, García y Guzmán (2016) encontraron que el trabajo del traspatio en la comunidad de Playa Ventura se divide entre los miembros de la familia, donde la mujer, además de otras tareas asignadas a su género -como la limpieza de la casa, la preparación de los alimentos y el cuidado de los hijos-es responsable de la organización y funcionamiento general, lo que se traduce en un ámbito de confianza y decisión en la realización de tareas productivas y en venta de productos como huevo y animales, o intercambio de animales o plantas con otras mujeres. 


\section{Contribución del programa PESA}

El programa PESA promueve el desarrollo de capacidades de los beneficiarios para el mejoramiento de la productividad, la innovación de los sistemas productivos y el manejo adecuado de los alimentos, con la intención de que la unidad familiar produzca sus propios alimentos y, cuando exista un excedente, los comercialice. Este programa enfatiza "la promoción y planeación participativa, con el propósito de identificar, formular, gestionar, poner en marcha y dar seguimiento a proyectos familiares que permitan contribuir a mejorar la salud en el hogar (a través del establecimiento de estufas ahorradoras de leña, silos para almacenar granos y sistemas de captación de agua de lluvia), producir alimentos de origen agrícola o pecuario para el autoconsumo y generar ingresos. Para ello, se plantea el objetivo de "desarrollar capacidades" en la población que vive en comunidades de alta marginación a través de agencias de desarrollo rural que promueven, de manera participativa, el desarrollo microregional por medio de proyectos y gestión local" (Cruz et al., 2016).

En los últimos años de "las solicitudes de apoyo recibidas en la SAGARPA para inscribirse en algunos de los programas, una de cada tres ha sido presentada por mujeres; en 2010 su participación creció en poco más del 50\%" (Zavala, 2011) y el programa PESA ha sido uno de ellos.

En las comunidades de estudio, la unidad familiar cuenta con aves y, de éstas, la gallina fue la principal porque se encontró en todos los traspatios; esto coincide con los estudios realizados por Monroy et al. (2016), Salazar et al. (2015), y Vieyra et al. (2004). Es la especie más común porque les proporciona alimento nutritivo, el manejo es muy sencillo, la inversión es poca y no requiere de mucho cuidado: "las gallinas casi se dan solas, ahí las dejas en el patio, les das poco maíz o tortilla, se suben a los árboles a dormir y así van creciendo" (Claudia Falfan Palma, comunicación personal, 2016).

Por lo anterior, las beneficiarias solicitaron apoyos para la producción de gallinas para hacer crecer su módulo, tecnificarlo y capacitarse más sobre el manejo de estas aves. La mayoría (60\%) de las encuestadas de las dos comunidades se enteraron de los apoyos del programa PESA a través de la difusión de las ADR, $23 \%$ mediante los vecinos, $10 \%$ por las autoridades locales y $7 \%$ en las instalaciones de las instituciones gubernamentales. Esto muestra la importante participación de las agencias en la difusión del programa.
La mitad de las encuestadas se organizó para gestionar el apoyo a través de reuniones grupales, $25 \%$ mediante la agencia de desarrollo que apoya a las comunidades, $20 \%$ trabajando en grupo y sólo $5 \%$ de forma individual. Respecto a la elaboración y entrega de trámites para obtener el apoyo fue fácil, 73\% opina que fue fácil, mientras que $27 \%$ dijo que fue regular.

En lo referente a las razones por las cuales solicitaron el apoyo, 55\% dijo para incrementar ingresos, 38\% para mejorar el manejo de las gallinas y $7 \%$ para poder vender y consumir los productos. Sin embargo, 100\% de las encuestadas considera que el apoyo ha contribuido a la mejora de sus ingresos debido a que adquieren excedentes del producto y pueden comercializarlo; además de que ya no gastan en la compra de huevo o de pollo, sino que pueden obtenerlos del traspatio y, de esta manera, tener una alimentación más nutritiva, segura de ser natural y fresca porque "las gallinas se crían y alimentan de manera natural sin muchos químicos, ya ve que ahora se dice que el huevo está bien viejo el que venden las empresas grandes" (Teresa Solís Riquemel, comunicación personal, 2016) (figura 3). Juárez et al. (2010) comentan que el huevo de gallinas de traspatio presenta dimensiones reducidas, en comparación con el huevo comercial. Sin embargo, los indicadores de calidad interna sugieren que la calidad del huevo de gallinas de traspatio es similar a la del huevo de gallinas de la estirpe comercial.

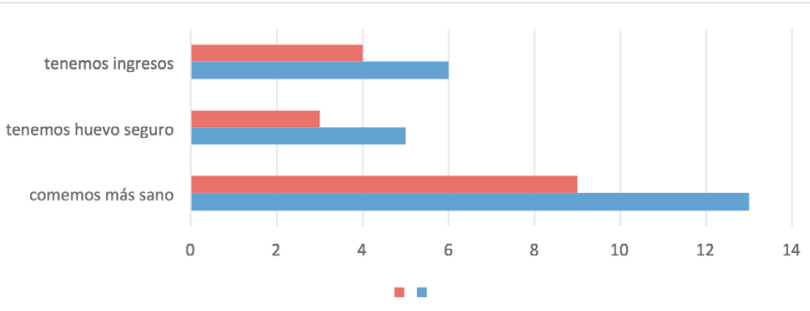

Figura 3. Percepción de las encuestadas sobre los beneficios adquiridos con el apoyo otorgado del PESA para el módulo de gallinas.

Aunque $77 \%$ de las encuestadas dijo que tener un módulo de gallinas ha incrementado el trabajo de la unidad doméstica porque tienen que ocuparse de la limpieza, manejo y alimentación de los animales, están contentas porque no es mucho lo que se hace: generan su propio empleo, obtienen ingresos para el sustento de la unidad familiar y "porque de cualquier manera tienen que trabajar y si adquieren una ganancia qué mejor" (Oliva Montesinos Sánchez, comunicación personal, 2016). Además, esta especie en el traspatio 
es común, ya que más de $80 \%$ de las familias rurales mantiene gallinas en sus viviendas (Santos et al., 2004). Se trata de un sistema tradicional de producción pecuaria que realiza la familia campesina en el patio de sus viviendas o alrededor de las mismas (Juárez et al., 2010).

Asimismo, 38\% de las beneficiarias opina que el programa es "muy bueno" y $42 \%$ "sólo bueno" porque con la producción del huevo pueden abastecer las carencias de este producto (autoconsumo), vender el excedente y adquirir otras necesidades básicas. Además, al generar su propio negocio, se sienten más seguras porque saben que tienen recursos para aportar a la unidad doméstica $\mathrm{y}$, de esta manera, pueden complementar los gastos; se sienten más integradas a las actividades de la comunidad porque ya no las ven solamente como amas de casa, sino que adquieren un estatus al participar en reuniones o cualquier actividad referente al programa; también generan lazos de amistad con otras participantes del programa porque durante el proceso de la gestión y ejecución del proyecto existe mucha convivencia: "ahora que tengo mis gallinas y me ha ido bien, empiezo a dar pláticas a otros lugares con otras personas que van a solicitar apoyo, a veces me invitan a la universidad a dar pláticas y yo me siento muy bien, mi autoestima ha crecido y sé que puedo hacer muchas cosas más, me siento contenta" (María Isabel Bueno Juárez, comunicación personal, 2016) (figura 4).

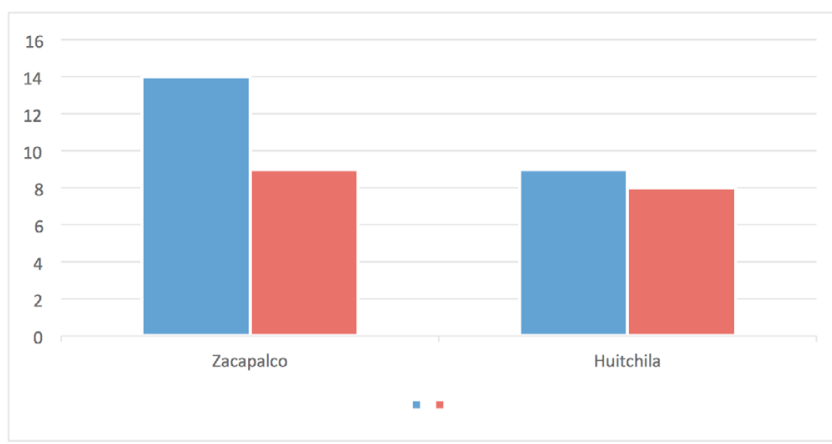

Figura 4. Beneficios adquiridos con el módulo de gallinas.

Las mujeres, en su labor cotidiana del hogar y del traspatio, reciben satisfactores tangibles e intangibles que les proporcionan comodidades, bienestar personal y familiar y empoderamiento (Vieyra et al., 2004; Rodríguez et al., 2011). Las oportunidades empresariales pueden dotar a las mujeres de independencia, mayor respeto y estatus social. Esto puede hacer que tengan una mayor participación en la toma de decisiones de su comunidad (FAO, 2007).
En contraste, $15 \%$ de las beneficiarias mencionó que el programa es regular y solamente $5 \%$ dijo que es malo porque las autoridades correspondientes no vigilan que los solicitantes del programa sean quienes realmente requieren el apoyo; tampoco existe un seguimiento en el que las autoridades revisen si las beneficiarias ciertamente utilizan el apoyo para lo que fue solicitado. Además, es importante supervisar a los proveedores con la entrega de lo cotizado porque en ocasiones no surten lo acordado, y, aun así, reciben su pago; por ello es sustancial que se les penalice o no reciban el pago correspondiente hasta que la entrega sea la correcta.

Cuando se les preguntó sobre cuál era su opinión respecto al programa, cinco de cada diez encuestadas dijeron que beneficiaba a la unidad doméstica, $23 \%$ mencionó que mejora los ingresos, $20 \%$ que programas de este tipo deben seguir apoyando a las familias y $7 \%$ opina que no deberían solicitar muchos requisitos porque en ocasiones se les dificulta conseguirlos. Todas opinaron que era un programa que apoyaba a las familias pero que debería tener mayor cobertura para que más unidades domésticas puedan beneficiarse.

Además, $70 \%$ dijo que no cambiaría nada al desarrollo y gestión del programa, ya que les parecía correcto, $18 \%$ mencionó que se deberían organizar más talleres para capacitarlas y $12 \%$ comentó que deberían eliminar la aportación del beneficiario, de manera que todo sea a fondo perdido.

No obstante, es transcendental que la participación de la mujer en este tipo de programas se incremente cada día porque, como sabemos, son ellas las responsables de la alimentación y cuidado de la unidad familiar, por lo que siempre estarán en busca de opciones para mejorar su calidad de vida, y qué mejor manera de hacerlo que en su propio hogar y comunidad. En relación con lo anterior, la FAO (2007) ha mencionado que el incremento del acceso de la mujer a oportunidades empresariales probablemente aumentará los ingresos domésticos y mejorará el bienestar y la nutrición en los hogares.

\section{Conclusiones}

En comunidades rurales de Morelos, el programa PESA es visto como un soporte económico importante: las gallinas son una especie apreciada por ser un ave de fácil manejo que, por tradición, se encuentra en el traspatio. La mayoría de las mujeres (55\%) solicita el recurso para incrementar la producción, consumir y vender $(7 \%)$ y para recibir capacitaciones 
sobre mejores técnicas de manejo (38\%). El 100\% de las beneficiaras mejoraron sus ingresos, ya sea a través de la venta de excedente del huevo o de la disminución de gastos al consumir lo que se produce. Por otro lado, $80 \%$ considera que los apoyos son muy importantes porque, con la producción del huevo, pueden abastecer las carencias de este producto (autoconsumo), vender el excedente y cubrir otras necesidades básicas. Además, perciben que producir el huevo en sus propios traspatios para autoconsumo les garantiza el acceso a un alimento fresco y natural.

Las mujeres, al hacerse cargo de una actividad que genera recursos y que contribuye a reducir los gastos de la unidad familiar, se consideran incluidas y valoradas en algunas decisiones que se deben tomar en la unidad familiar y, en ocasiones, en la comunidad. Participar en las actividades organizadas para las beneficiaras del apoyo les permite generar lazos de amistad y aprender diferentes temas que les enseñan los profesionistas de las agencias, lo que les origina mayor seguridad y autoestima, e incluso las motiva a participar más activamente en todo el proceso.

La implementación de estos programas es muy importante, por lo que es necesario elaborar estrategias donde se beneficie a las familias de pequeños productores para que incrementen su producción de manera sustentable y se los capacite durante toda la cadena de valor de su producción y en cómo llevar una nutrición adeciada.

\section{Agradecimientos}

A las familias de las localidades de Huitchila y Zacapalco, Tepalcingo, Morelos, quienes nos abrieron sus puertas y su confianza para realizar las encuestas y entrevistas, así como al programa de apoyo Fortalecimiento de Cuerpos Académicos de PRODEP. 


\section{LiterATURA CITADA}

Adebayo, O. O. \& R. G. Adeola. 2005. Socio-economics factors affecting poultry farmers in Ejigbo local government area of Osun state. Human Ecology 18(1): 39-41.

Álvarez, J. L. 2003. Cómo Hacer Investigación Cualitativa. Fundamentos y Metodologías. Paidós Ecuador. México, D.F. 222 pp.

Bonfil, G. 1991. Pensar Nuestra Cultura. Alianza Editorial. México, D.F. 172 pp.

Bonilla, M. E., B. A. Salcido, J. A. Paredes, L. Aguirre, M. E. Méndez \& M. L. Hernández. 2013. La diversidad hortícola para la seguridad alimentaria en municipios marginados del estado de Puebla. Ra Ximhai 9(2): 151-163.

Carbonell, M. 2015. Constitución Política de los Estados Unidos Mexicanos. Tirant lo Blanch. México. 175 pp.

Chiriboga, M. 1997. Desafíos de la pequeña agricultura familiar frente a la globalización. Perspectivas Rurales 1: 9-24.

Colín, H., A. Hernández \& R. Monroy. 2012. El manejo tradicional y agroecológico en un huerto familiar de México, como ejemplo de sostenibilidad. Etnobiología 10(2): 12-28.

Cruz, B., M. Muñoz, V. H. Santoyo, E. G. Martínez \& N. Aguilar. 2016. Potencial y restricciones de la avicultura de traspatio sobre la seguridad alimentaria en Guerrero, México. Agricultura, Sociedad y Desarrollo 13: 257-275.

Emaikwu, K. K, D. O. Chikwendu \& A. S. Sani. 2011. Determinants of flock size in broiler production in Kaduna State of Nigeria. Agricultural Extension and Rural Development 3(11): 202-211.

Espinosa, L. \& S. Diez- Urdanivia. 2006. Notas sobre la contribución de la mujer a la seguridad alimentaria de la unidad doméstica campesina. Nueva Antropología 20(066): 11-31.

García, A. \& E. Guzmán. 2016. El guajolote nativo, elemento cotidiano del traspatio en Playa Ventura, Copala, Guerrero, México. Agricultura, Sociedad y Desarrollo 13(1): 1-18.

Gimate, S. A. 2016. Análisis basado en la evidencia de seguridad alimentaria: PESA-Oaxaca, México. Ciencias Sociales 4(154):129-148.

González, H. 2007. La gobernanza mundial y los debates sobre la seguridad alimentaria. Desacatos 25: 7-20.

González, F., A. Pérez, I. Ocampo, J. A. Paredes \& P. De la Rosa. 2014. Contribuciones de la producción en traspatios a los grupos domésticos campesinos. Estudios Sociales 22(44): 145-170.

Guarneros, N., J. Morales, J. Cruz, A. Huerta \& D. A. Dávalos. 2014. Economía familiar e índice de diversidad de especies en los traspatios comunitarios de Santa María Nepopualco, Puebla. Mexicana de Ciencias Agrícolas 9:1701-1712.

Guerrero, A. G. 2007. El impacto de la migración en el manejo de solares campesinos, caso de estudio La Purísima Concepción Mayorazgo, San Felipe del Progreso, Estado de México. Investigaciones Geográficas 63: 105-124.
Guzmán, E. 2005. Resistencia, Permanencia y Cambio. Estrategias Campesinas de Vida en el Poniente de Morelos. Plaza y Valdés-UAEM. Cuernavaca, México. 314 pp.

Guzmán, E. 2004. Mujeres, trabajo y organización familiar: los traspatios en Ahuehuetzingo, Morelos. pp. 1-49. In Suárez, B. Bonfil, P. (Coords.). Entre el corazón y la necesidad. Microempresas familiares en el medio rural. Gimtrap-PEMSA, México.

Hernández, R., C. Fernández \& P. Baptista. 2006. Metodología de la Investigación. Mc Graw Hill-Interamericana. México, D.F. 850 pp.

Houtart, F. 2010. La agroenergía: sus dimensiones socioeconómicas. Entre las crisis alimentaria, climática y de capital. Ciencias Sociales y Humanas 12: 47-85.

Instituto Interamericano de Cooperación para la Agricultura (IICA). 2012. Situación de la Seguridad Alimentaria en las Américas. Documento para Alimentar el Dialogo de la $42^{\mathrm{a}}$ Asamblea General de la Organización de los Estados Americanos. San José, Costa Rica.

Instituto Nacional de Estadísticas y Geografía (INEGI). 2010. Sistema Nacional de Información Estadística y Geográfica. Sistema para la Consulta del Anuario Estadístico Morelos.

Juárez, A., E. Gutiérrez, J. Segura \& R. Santos. 2010. Calidad del huevo de gallinas criollas criadas en traspatio en Michoacán México. Tropical and Subtropical Agroecosystems 12(1): 109-115.

Landini, F. 2012. Problemas en la extensión rural paraguayana: modelos de extensión en la encrucijada. Cuadernos de Desarrollo Rural 9: 127-149.

Lobo, L. 2014. Contexto regional: las trampas de la lucha contra la desnutrición crónica infantil. pp. 79-92. In: FAO (Eds.). cooperación internacional y políticas públicas de seguridad alimentaria y nutricional. La experiencia del programa España-FAO para América Latina y el Caribe. FAO.

López, J. L., M. A. Damián, F. Álvarez, G. P. Zuluaga, F. Parra \& J. A. Paredes. 2013. El traspatio de los productores de maíz en San Nicolás de los Ranchos, Puebla-México. Ra Himhai 9(2): 181- 198.

Martínez, E. G., M. Muñoz, V. H. Santoyo, D. Gómez \& R. Altamirano. 2013. Lecciones de la promoción de proyectos de caprinos a través del programa estratégico de seguridad alimentaria en Guerrero, México. Agricultura, Sociedad y Desarrollo 10(2): 177-193.

Monje, C. A. 2011. Metodología de la Investigación Cuantitativa y Cualitativa Guía Didáctica. Universidad Surcolombiana. Colombia. 216 pp.

Monroy, R., A. Ponce, H. Colín, C. Monroy \& A. García. 2016. Ambiente y Sostenibilidad 6: 33-43.

Obidzinski, K., R. Andriani, H. Komarudin \& A. Andrianto, 2012. Environmental and social impacts of oil palm plantations and their implications for biofuel production in Indonesia. Ecology and Society 17(1): 25. 
Ordóñez, F. 2010. La agroecología y la soberanía alimentaria como alternativa al sistema agroalimentario capitalista. Experiencia de la Fundación San Isidro (Duitama, Colombia). IILSA 42: 203-247.

Organización de las Naciones Unidas para la Agricultura y la Alimentación (FAO). 1996. Cumbre Mundial sobre la alimentación. 13-17 de noviembre. Roma, Italia.

Organización de las Naciones Unidas para la Agricultura y la Alimentación (FAO). 2007. El Estado Mundial de la Agricultura y la Alimentación. FAO- Agricultura 38, Italia. $255 \mathrm{pp}$.

Organización de las Naciones Unidas para la Agricultura y la Alimentación (FAO). 2011. La Seguridad Alimentaria: Información para la toma de decisiones. CE-FAO, Italia.

Organización de las Naciones Unidas para la Agricultura y la Alimentación (FAO). 2015. El estado de la Inseguridad alimentaria en el mundo 2015: cumplimiento de los objetivos internacionales para 2015 en relación con el hambre: balance de los desiguales progresos. Organización de las Naciones Unidas para la Agricultura y la Alimentación (FAO), Fondo Internacional de Desarrollo Agrícola (FIDA) \& Programa Mundial de Alimentos (PMA). Italia. 66 pp.

Oseguera, D. \& L. L. Esparza. 2009. Significados de la seguridad y el riesgo alimentarios entre indígenas purhépechas de México. Desacatos 31: 115-136.

Proyecto Estratégico de Seguridad Alimentaria- Secretaría de Agricultura, Ganadería, Desarrollo Rural, Pesca y Alimentación (PESA-Sagarpa). 2017. Requisitos documentos programa Sagarpa.

Riat, P. \& M. L. Pochettino. 2014. ¿Para usar o eliminar? El uso local del Monte Santiagueño (Argentina) y el avance de la agricultura industrial. Zonas Áridas 15(1): 68-91.

Rodríguez, G., R. Perezgrovas \& L. Zaragoza. 2011. El traspatio como espacio de empoderamiento para la mujer Tzotzil en Chiapas, México. Actas Iberoamericanas de Conservación Animal 1: 280-283.

Rodríguez, G., J. Gil \& E. García. 1996. Metodología de la Investigación Cualitativa. Editorial Aljibe. Málaga, España. 380 pp.

Román, E. 2016. La Milpa Amatleca como Estrategia de Vida. Universidad Autónoma del Estado de México. Cuernavaca, México. 204 pp.

Román, E. \& E. Guzmán. 2013. Mujer, trabajo y persistencia del maíz. Estudios de Género La Ventana 4 (38): 164211.

Rubio, B. 2013. La crisis alimentaria en México. pp. 53-83. In Rubio, B. (Coord.). La crisis alimentaria mundial impacto sobre el campo mexicano. UNAM-Miguel Ángel Porrúa, México.

Salah, O. M., F. L. Sarmiento, J. F. J. Torres \& L. Fernández. 2017. Understanding the information acquisition sources of backyard chicken production practices amongst rural Mayan women in Yucatán, México. Agricultura, Sociedad y Desarrollo 14(2): 219-237.
Salazar, L. L., M. A. Magaña \& L. Latornerie. 2015. Importancia económica y social de la agrobiodiversidad del traspatio en una comunidad rural de Yucatán México. Agricultura, Sociedad y Desarrollo 12(1): 1-14.

Santos, R., C. E. Hau, R. Belmar, I. Armendáriz, R. Cetina, J. Sarmiento \& J. C. Segura. 2004. Socio-economic and technical characteristics of backyard animal husbandry in two rural communities of Yucatan, Mexico. Agriculture and Rural Development in the Tropics and Subtropics 105(2): 165-173.

Servicio de Información Agropecuaria Pesquera (SIAP) Secretaría de Agricultura, Ganadería, Desarrollo Rural, Pesca y Alimentación (SAGARPA). 2015. Producción Agropecuaria, México.

Suárez, M. H. 2001. Rezago Educativo y desigualdad social en el estado de Morelos. Retos de la gestión social. UNAM-CRIM-Miguel Ángel Porrúa, México. 78 pp.

Verduzco, C., E. G. Martínez, M. Muñoz, V. H. Santoyo \& J. Aguilar. 2016. Estrategia de gestión de la innovación para la avicultura de traspatio en zonas rurales marginadas de Oaxaca, México. Turismo, Economía y Negocios Transitare 2(2): 165-182.

Vieyra, J., A. Castillo, H. Losada, J. Cortés, G. Alonso, T. Ruíz, P. Hernández, A. Zamudio \& A. Acevedo. 2004. La participación de la mujer en la producción traspatio y sus beneficios tangibles e intangibles. Cuadernos del Desarrollo Rural 53: 9-23.

Zavala, L. 2011. Incentiva México la participación de la mujer en el medio rural. Unión campesina. Disponible en: http://ucd.org.mx/ (consultado: 1 de julio de 2017). 
Anexo 1. Cuestionario socioeconómico.

\section{CUESTIONARIO SOCIECONÓMICO}

1. ¿Cuál es su nombre?

2. ¿De donde son originarios?

3. ¿Cuanto tiempo llevan viviendo en la localidad?

4. Casa: Propia ( ) Renta ( ) Prestada ( ) Otros (especifique):

5. Integrantes de la familia

\begin{tabular}{|l|l|l|l|l|l|l|l|l|}
\hline EDAD & SEXO & NOMBRE & PARENTESCO & ESCOLARIDAD & OCUPACION & $\begin{array}{l}\text { EDO } \\
\text { CIVLL }\end{array}$ & $\begin{array}{l}\text { NGRESOI } \\
\text { MES }\end{array}$ & $\begin{array}{l}\text { MIGRANTES } \\
\text { ADONOE }\end{array}$ \\
\hline & $\mathrm{F}$ & $\mathrm{M}$ & & & & & & \\
\hline & & & & & & & & \\
\hline & & & & & & & & \\
\hline & & & & & & & & \\
\hline & & & & & & & & \\
\hline & & & & & & & \\
\hline
\end{tabular}

6. Tipo de casa (OBSERVACIÓN).

( ) Casa individual

( ) Techo sin paredes y patio

( )Apartamento

( ) Habitación dentro de casa

( ) Otro

7. ¿Qué material de construcción se usó para la MAYORIÁ de los MUROS exteriores de la casa o edificio?

\begin{tabular}{|l|}
\hline ( ) Bloque/ladrillo/piedra/cemento \\
( ) Lamina de cartón \\
( ) Madera \\
( ) Adobe/caña y barro \\
( ) Caña/paja/palos \\
( ) Otro \\
\hline
\end{tabular}

8. ¿Cuál es el material de construcción de la mayor parte del techo de ésta casa?

( ) Concreto/cemento

( ) Tejas

( ) Fibra/Plástico

( ) Metal (zinc, aluminio, etc.)

( ) Madera

( ) Paja

( ) Lamina de Asbesto

( ) Otro

9. ¿Cuál es el material de construcción de la mayor parte del piso de ésta casa?

( ) Concreto/cemento

( ) Mosaico, ladrillo, granito

( ) Madera

( ) Vinil/Plástico

( ) Tierra, arena

( ) Otro

10. ¿Cuántas habitaciones hay en la casa?

11. ¿Cuántas habitaciones se emplean exclusivamente para dormir?

12. ¿Que clase de servicios sanitarios utiliza este hogar?

( ) Drenaje

( ) Fosa séptica

( ) Letrina o baño seco

( ) Ninguno 
13. ¿Cuál es la fuente primordial de agua para este hogar?

\begin{tabular}{|l|}
\hline ( ) Sistema público \\
( ) Pozo o cisterna propia \\
( ) Toma abierta \\
( ) Manantiales u ojos de agua \\
( ) Otro
\end{tabular}

14. ¿Cómo se deshace este hogar de la mayoria de su basura?

( ) Recolección pública (conteneros)

( ) Tirar en lotes baldios

( ) Tirar en patio anterior

( ) La tira en la barranca

( ) Quemar o enterrar

( ) Otro

15. ¿Qué tipo de iluminación utiliza este hogar?

( ) Electricidad (pública)

( ) Otros (gas, velas)

16. ¿Cuenta con traspatio?

( ) Si ¿Desde cuándo? Años ( )

( ) no

17. ¿Qué tipo de animales y cuántos tiene en el traspatio?

18. ¿Qué tipo de plantas tiene en el traspatio y cuáles son?

19. ¿Qué otras especies tiene en el traspatio?

20. ¿Quién se hace carqo del cuidado del traspatio? 


\section{CUESTIONARIO SOBRE APOYO DEL PROYECTO ESTRATÉGICO PARA LA SEGURIDAD ALIMENTARIA (PESA)}

La productora puede estar segura de que la información que proporcione será absolutamente confidencial y utilizada sólo con fines de estudio.

1. ¿Cómo se enteró del Programa?

a. Por difusión (radio, televisión, tripticos, platica por parte de la Sedagro)

b. En las instalaciones de SAGARPA o Sedagro

c. Por vecinos

d. Autoridades de la localidad

e. Otro (especifique)

2. ¿Cuál es su opinión acerca del Programa PESA?

3. ¿De qué manera se organizaron para gestionar el apoyo?

4. ¿De qué manera gestionó el apoyo?

5. ¿Cuál es su opinión acerca de los trámites que se requieren para obtener el apoyo?

6. ¿En qué año recibió el apoyo?

7. ¿Por qué solicitó el apoyo?

8. ¿Qué recibió por parte del programa?
a. Animales
Cuántos ( )
b. Plantas
Cuántas ( )
c. Material de construcción
d. Infraestructura
e. Maquinaria y equipo
f. Otro (especifique)

8. ¿Cuánto recurso puso el Programa PESA y cuánto Ud.?

9. ¿Considera que el apoyo del Programa PESA ha mejorado su ingreso económico?

( ) $\mathrm{Si}$

( ) No

¿Por qué?

10. ¿El apoyo del Programa PESA ha mejorado su alimentación?

( ) $\mathrm{Si}$

¿Por qué?

11. ¿Cree que este Programa ayuda a que las familias tengan una mejor alimentación?

( ) $\mathrm{Si}$

No

¿Por qué?

12. ¿El apoyo del programa considera que puede ayudar a que las familias tengan mayores ingresos con la producción?

( ) $\mathrm{Si}$

() No

¿Por qué? 
13. ¿Considera que el apoyo del programa PESA le ha generado mayor trabajo fisico?

( ) $\mathrm{Si}$

( ) No

¿Por qué?

14. ¿Continúa utilizando el apoyo del programa para producir algún producto?

( ) $\mathrm{Si}$

( ) No

¿Por qué?

15. ¿Cuál es su opinión acerca del programa PESA?

16. ¿Qué le cambiaría al programa PESA?

17. ¿Ha recibido algún tipo de capacitación para la producción de su producto?

( ) $\mathrm{Si}$

( ) No

¿Cuál?

18. ¿Le dieron asesoría al inicio y durante la producción del producto?

( ) Si inicio ( ) durante la producción ( ) siempre ( )

( ) No

19. ¿Ha sido invitada a participar en reuniones para opinar sobre el programa y mejorarlo? ¿Cuáles?

20. Algún comentario que quiera aportar 
Anexo 2. Características educativas de algunas comunidades de Tepelcingo, Morelos.

\begin{tabular}{|c|c|c|c|c|c|c|c|c|c|c|c|c|}
\hline \multicolumn{13}{|c|}{ Caracteristicas educativas de algunas comunidades de Tepalcingo Morelos } \\
\hline \multirow{2}{*}{ Descripción } & Huitchila & Zacapalco & Pitzotlán & $\begin{array}{l}\text { Los } \\
\text { Sauces }\end{array}$ & $\begin{array}{c}\text { EI } \\
\text { Tepehuaje }\end{array}$ & $\begin{array}{l}\text { Cruz de } \\
\text { Jaramalla }\end{array}$ & Huitchila & Zacapalco & Pitzotlán & $\begin{array}{c}\text { Los } \\
\text { Sauces }\end{array}$ & $\begin{array}{c}\text { EI } \\
\text { Tepehuaje }\end{array}$ & $\begin{array}{l}\text { Cruz de } \\
\text { Jaramalla }\end{array}$ \\
\hline & \begin{tabular}{|c|}
1935 \\
habitante
\end{tabular} & \begin{tabular}{|c|c|} 
& 1714 \\
habitantes
\end{tabular} & $\begin{array}{c}39 \\
\text { habitantes }\end{array}$ & \begin{tabular}{|c|}
298 \\
habitantes
\end{tabular} & \begin{tabular}{|c|}
136 \\
5 \\
habitantes
\end{tabular} & \begin{tabular}{|c|}
337 \\
habitantes
\end{tabular} & \multicolumn{6}{|c|}{$\begin{array}{l}\text { Proporción No. de habitantes entre descripción } \\
\qquad \%\end{array}$} \\
\hline $\begin{array}{l}\text { Población de } 3 \text { a } 5 \text { años que no } \\
\text { asiste a la escuela }\end{array}$ & 86 & 57 & 2 & 5 & 6 & 12 & 4.44 & 3.33 & 5.13 & 1.68 & 4.41 & 3.5 \\
\hline $\begin{array}{l}\text { Población masculina de } 3 \text { a } 5 \\
\text { años que no asiste a la escuela }\end{array}$ & 48 & 23 & 2 & 3 & 3 & 7 & 2.48 & 1.34 & 5.13 & 1.01 & 2.21 & 2.08 \\
\hline $\begin{array}{l}\text { Población femenina de } 3 \text { a } 5 \\
\text { años que no asiste a la escuela }\end{array}$ & 38 & 34 & 0 & 2 & 3 & 5 & 1.96 & 1.98 & 0.00 & 0.67 & 2.21 & 1.48 \\
\hline $\begin{array}{l}\text { Población de } 6 \text { a } 11 \text { años que no } \\
\text { asiste a la escuela }\end{array}$ & 10 & 8 & 0 & 0 & 1 & 1 & 0.52 & 0.47 & 0.00 & 0.00 & 0.74 & 0.30 \\
\hline \begin{tabular}{|l|} 
Población masculina de 6 a 11 \\
años que no asiste a la escuela
\end{tabular} & 5 & 6 & 0 & 0 & 1 & 1 & 0.26 & 0.35 & 0.00 & 0.00 & 0.74 & 0.30 \\
\hline \begin{tabular}{|l|} 
Población femenina de 6 a 11 \\
años que no asiste a la escuela
\end{tabular} & 5 & 2 & 0 & 0 & 0 & 0 & 0.26 & 0.12 & 0.00 & 0.00 & 0.00 & 0.00 \\
\hline $\begin{array}{l}\text { Población de } 12 \text { a } 14 \text { años que } \\
\text { no asiste a la escuela }\end{array}$ & 16 & 9 & 0 & 1 & 2 & 3 & 0.83 & 0.53 & 0.00 & 0.34 & 1.47 & 0.89 \\
\hline $\begin{array}{l}\text { Población masculina de } 12 \text { a } 14 \\
\text { años que no asiste a la escuela }\end{array}$ & 8 & 5 & 0 & 0 & 2 & 1 & 0.41 & 0.29 & 0.00 & 0.00 & 1.47 & 0.30 \\
\hline $\begin{array}{l}\text { Población femenina de } 12 \text { a } 14 \\
\text { años que no asiste a la escuela }\end{array}$ & 8 & 4 & 0 & 1 & 0 & 2 & 0.41 & 0.23 & 0.00 & 0.34 & 0.00 & 0.59 \\
\hline $\begin{array}{l}\text { Población de } 8 \text { a } 14 \text { años que no } \\
\text { saben leer y escribir }\end{array}$ & 20 & 13 & 1 & 0 & 1 & 2 & 1.03 & 0.76 & 2.56 & 0.00 & 0.74 & 0.59 \\
\hline $\begin{array}{l}\text { Población masculina de } 8 \text { a } 14 \\
\text { años que no saben leer y } \\
\text { escribir }\end{array}$ & 10 & 11 & 1 & 0 & 1 & 1 & 0.52 & 0.64 & 2.56 & 0.00 & 0.74 & 0.30 \\
\hline $\begin{array}{l}\text { Población femenina de } 8 \text { a } 14 \\
\text { años que no saben leer y } \\
\text { escribir }\end{array}$ & 10 & 2 & 0 & 0 & 0 & 1 & 0.52 & 0.12 & 0.00 & 0.00 & 0.00 & 0.30 \\
\hline $\begin{array}{l}\begin{array}{l}\text { Población de } 15 \text { años y más } \\
\text { analfabeta }\end{array} \\
\end{array}$ & 170 & 155 & 5 & 40 & 21 & 20 & 8.79 & 9.04 & 12.82 & 13.42 & 15.44 & 5.93 \\
\hline $\begin{array}{l}\text { Población masculina de } 15 \text { años } \\
\text { y más analfabeta }\end{array}$ & 84 & 72 & 2 & 30 & 11 & 6 & 4.34 & 4.20 & 5.13 & 10.07 & 8.09 & 1.78 \\
\hline $\begin{array}{l}\text { Población femenina de } 15 \text { años } \\
\text { y más analfabeta }\end{array}$ & 86 & 83 & 3 & 10 & 10 & 14 & 4.44 & 4.84 & 7.69 & 3.36 & 7.35 & 4.15 \\
\hline $\begin{array}{l}\text { Población de } 15 \text { años y más sin } \\
\text { escolaridad }\end{array}$ & 152 & 174 & 4 & 22 & 14 & 27 & 7.86 & 10.15 & 10.26 & 7.38 & 10.29 & 8.01 \\
\hline $\begin{array}{l}\text { Población masculina de } 15 \text { años } \\
\text { y más sin escolaridad }\end{array}$ & 77 & 92 & 2 & 13 & 8 & 9 & 3.98 & 5.37 & 5.13 & 4.36 & 5.88 & 2.67 \\
\hline $\begin{array}{l}\text { Población femenina de } 15 \text { años } \\
\text { y más sin escolaridad }\end{array}$ & 75 & 82 & 2 & 9 & 6 & 18 & 3.88 & 4.78 & 5.13 & 3.02 & 4.41 & \\
\hline
\end{tabular}

